PROCEEDINGS OF THE

AMERICAN MATHEMATICAL SOCIETY

Volume 131, Number 12, Pages 3861-3871

S 0002-9939(03)07000-X

Article electronically published on May 8, 2003

\title{
ON THE EMBEDDING OF VARIATIONAL INEQUALITIES
}

\author{
B. DJAFARI ROUHANI AND A. A. KHAN
}

(Communicated by Joseph A. Ball)

Dedicated to Jochem Zowe on the occasion of his sixtieth birthday

\begin{abstract}
This work is devoted to the approximation of variational inequalities with pseudo-monotone operators. A variational inequality, considered in an arbitrary real Banach space, is first embedded into a reflexive Banach space by means of linear continuous mappings. Then a strongly convergent approximation procedure is designed by regularizing the embedded variational inequality. Some special cases have also been discussed.
\end{abstract}

\section{INTRODUCTION}

Throughout the paper, unless the contrary is stated, $Y$ is a real Banach space, $Y^{*}$ its topological dual and $\langle\cdot, \cdot\rangle_{Y}$ the associated pairing. Let $\|\cdot\|_{Y}$ be the norm in $Y$ as well as in $Y^{*}$. Let $\Omega \subset Y$ be nonempty, closed and convex. Consider a single-valued operator $F: D(F) \subseteq Y \rightarrow Y^{*}$ and let $f \in Y^{*}$ be arbitrary. Here and throughout, $D(F)$ will be the effective domain of $F$. The symbols " $\rightarrow$ " and "-" are used to specify the strong and the weak (weak*) convergence, respectively.

We consider the following problem: find $y \in \Omega$ such that

$$
\langle F y-f, x-y\rangle_{Y} \geq 0, \quad \forall x \in \Omega .
$$

The above problem is the celebrated Variational Inequality (for short, $V I$ ) introduced by G. Stampacchia (see [11]). Any element $y \in \Omega$ satisfying the above conditions is said to be a solution to $V I(1)$. We shall denote by $S(V I)$ the set of all solutions to $V I(1)$.

If $\Omega$ is a closed and convex cone with apex at the origin, then $V I(\mathbb{1})$ collapses to the following Nonlinear Complementarity Problem (for short, $N C P$ ): find $y \in Y$ such that

$$
y \in \Omega, \quad F y-f \in \Omega^{*}, \quad\langle F y-f, y\rangle_{Y}=0,
$$

where $\Omega^{*}$ denotes the (positive) polar of $\Omega$ (for details on $N C P$ see for instance 8]).

In recent years the theory of $V I$ and $N C P$ has emerged as an important branch of pure, applied and industrial mathematics (see [3], [4, 7], 11], [17]).

Received by the editors October 22, 2001 and, in revised form, August 1, 2002.

2000 Mathematics Subject Classification. Primary 47A52; Secondary 47H14.

Key words and phrases. Variational inequalities, regularization, pseudo-monotone, embedding.

The first author's research was supported by a grant from Shahid Beheshti University.

The second author's research was supported by the German Science Foundation (DFG). 
Let us assume that instead of the exact data $(F, f, \Omega)$ for $V I(\mathbb{1})$, only perturbed data $\left(F_{n}, f_{n}, \Omega_{n}\right)$ are available. Throughout, it will be assumed that $\forall n \in \mathbb{N}, f_{n} \in$ $Y^{*}$ and $\Omega_{n}$ are nonempty closed and convex. The relationship between $(F, f, \Omega)$ and $\left(F_{n}, f_{n}, \Omega_{n}\right)$ will be made precise shortly.

Therefore, we need to deal with the following Perturbed VI (for short, PVI):

$$
\text { find } y \in \Omega_{n} \text { such that }\left\langle F_{n} y-f_{n}, x-y\right\rangle_{Y} \geq 0, \quad \forall x \in \Omega_{n} .
$$

The aim of the present work is to develop a stable approximation procedure for the above problem. However, the strategy we adopt here for the stable approximation of PVI (2) differs from the standard stabilization procedures (for a comparison see 11, [15]). Since it is well known that PVI(21) is in general ill-posed, a regularization procedure is incorporated, however, contrary to the usual approach, the regularization is carried out in an arbitrary space fulfilling certain requirements. For the purpose, $V I(2)$ is first embedded into another space and the rest of the treatment is done in the topology of this new space. One advantage of this approach is that we impose minimal restrictions on the space $Y$, in which $V I(\mathbb{1})$ is posed. For example, the space $Y$ need not be reflexive. It is known that the regularization of minimization problems in nonreflexive spaces is crucial for classical problems such as the minimal surface problem and for modern problems such as image reconstruction (see [16]). Since a variational inequality is a necessary optimality condition for minimization problems our results are also applicable to such problems. Our approach resembles the so-called Elliptic-super-regularization, introduced by Browder-Ton [2] (see also Ton [18]). However, the idea of using the topology of the space in which the $V I$ is embedded was originated in [9] for variational inequalities with pseudo-monotone operators (in the sense of Karamardian) and later developed in 10] for a more general setting. In this paper, we extend and refine the earlier results (cf. [9], [10]) for pseudo-monotone operators (in the sense of H. Brézis).

The rest of the paper is organized as follows: In the next section, we recall some results and concepts. Section 3 begins with certain technical results. These results are further used to define a regularized form of $P V I(2)$. We show that the regularized problem is strongly convergent. The paper concludes with some remarks concerning the approach.

\section{Preliminaries}

Let $Z$ be a real reflexive Banach space, let $Z^{*}$ be the topological dual of $Z$, let $\langle\cdot, \cdot\rangle_{Z}$ be the associated pairing and let $\|\cdot\|_{Z}$ be the norm in $Z$.

The following notion of a pseudo-monotone operator was introduced by H. Brézis (cf. 20]).

Definition 2.1. Let $T: D(T) \subseteq Z \rightarrow Z^{*}$. The operator $T$ is said to be pseudomonotone, if for $x_{n}, x \in D(T)$ such that $x_{n} \rightarrow x$ together with

$$
\limsup _{n \rightarrow \infty}\left\langle T x_{n}, x_{n}-x\right\rangle_{Z} \leq 0
$$

imply

$$
\liminf _{n \rightarrow \infty}\left\langle T x_{n}, x_{n}-z\right\rangle_{Z} \geq\langle T x, x-z\rangle_{Z}, \quad \forall z \in D(T) .
$$

Definition 2.2. Let $T: D(T) \subseteq Z \rightarrow Z^{*}$. (i) The operator $T$ is said to be demicontinuous, if for $x \in D(T)$, and any sequence $x_{n} \rightarrow x$ with $x_{n} \in D(T)$, we 
have $T x_{n} \rightarrow T x$. (ii) The operator $T$ is said to be hemicontinuous, if for $x_{0} \in D(T)$, and for any vector $x$ such that $x_{0}+t x \in D(T)$ for $0 \leq t \leq \alpha(\alpha=\alpha(x)>0)$ and for any sequence $t_{n} \rightarrow 0$ as $n \rightarrow \infty\left(0<t_{n} \leq \alpha\right)$, we have $T\left(x_{0}+t_{n} x\right) \rightarrow T x_{0}$.

It is known that if $T: Z \rightarrow Z^{*}$ is monotone and hemicontinuous, then $T$ is pseudo-monotone and that the sum of two pseudo-monotone operators is pseudomonotone.

Now we recall the so-called Mosco convergence of convex sets (see Mosco [14]).

Definition 2.3. Let $K_{\rho}$ be a sequence of nonempty, closed and convex sets. The sequence $K_{\rho}$ is said to converge to a set $K$ as $\rho$ approaches zero, denoted by $K_{\rho} \rightrightarrows K$, if and only if the following two conditions hold:

[M1]: If $u_{\rho} \in K_{\rho}$ where $\rho$ is a sequence which converges to zero and $u_{\rho} \rightarrow u$, then $u \in K$.

$[M 2]$ : For each $u \in K$ there exists a $u_{\rho} \in K_{\rho}$ such that $u_{\rho} \rightarrow u$.

The proof of the following result can be obtained from Zeidler [20].

Theorem 2.1. Let $K \subseteq Z$ be nonempty, closed and convex and let $T: K \rightarrow Z^{*}$ be bounded, demicontinuous and pseudo-monotone. Let $f \in Z^{*}$ be an arbitrary element. Assume that the operator $T$ is coercive in the following sense: there exists $x_{0} \in K$ such that $\left\langle T x, x-x_{0}\right\rangle_{Z} /\|x\|_{Z} \rightarrow+\infty$ as $\|x\|_{Z} \rightarrow+\infty$. Then, there exist at least one $y \in K$ such that

$$
\langle T y-f, x-y\rangle_{Z} \geq 0, \quad \forall x \in K .
$$

\section{Regularization}

Let $X$ be a real reflexive Banach space and let $X^{*}$ be its topological dual. Let $\langle\cdot, \cdot\rangle_{X}$ be the associated pairing and let $\|\cdot\|_{X}$ be the norm in $X$ as well as in $X^{*}$.

Let $\zeta$ be a single-valued mapping defined from $X$ to $Y$. Throughout the present work, it will be assumed that $\zeta$ is linear and continuous and the sets $\Pi:=\Omega \cap \zeta(X)$ and $\Pi_{n}:=\Omega_{n} \cap \zeta(X)$ are nonempty. We use the notation $\zeta^{*}$ to specify the transpose of the mapping $\zeta$ which is defined from $Y^{*}$ to $X^{*}$.

Consider the following Regularized VI (for short, $R V I$ ): find $x_{n} \in \zeta^{-1}\left(\Pi_{n}\right)$ such that for all $z \in \zeta^{-1}\left(\Pi_{n}\right)$ we have

$$
\left\langle\zeta^{*} F_{n} \zeta x_{n}+\epsilon_{n} R x_{n}-\zeta^{*} f_{n}, z-x_{n}\right\rangle_{X} \geq-\epsilon_{n} \kappa\left(\left\|\zeta x_{n}\right\|_{Y}\right)\left\|\zeta z-\zeta x_{n}\right\|_{Y},
$$

where $R: X \rightarrow X^{*}$ is hemicontinuous and strongly monotone, $\kappa: \mathbb{R}_{+} \rightarrow \mathbb{R}_{+}$is nondecreasing and bounded and $\epsilon_{n}>0$.

Recall that $R$ is said to be strongly monotone iff there exists a constant $m>0$ such that

$$
\langle R(x)-R(z), x-z\rangle \geq m\|x-z\|^{2}, \quad \forall x, y \in X .
$$

We shall denote by $S_{n}(R V I)$ the set of all solutions to $R V I(3)$.

In order to justify the formulation of $R V I(3)$ we give some auxiliary results.

Lemma 3.1. For $n \in \mathbb{N}$, the sets $\zeta^{-1}\left(\Pi_{n}\right)$ and $\zeta^{-1}(\Pi)$ are closed and convex subsets of $X$.

Proof. We prove the assertion for $\zeta^{-1}\left(\Pi_{n}\right)$. For the purpose, let $n \in \mathbb{N}$ be arbitrary. As $\zeta$ is a single-valued mapping form $X$ to $Y, \zeta^{-1}\left(\Pi_{n}\right)$ is a set in $X$. Convexity of this set follows as a consequence of convexity of $\Omega_{n}$ and linearity of the single-valued mapping $\zeta$. For closedness, let $x_{k} \in \zeta^{-1}\left(\Pi_{n}\right)$ with $x_{k} \rightarrow x$. We claim $x \in \zeta^{-1}\left(\Pi_{n}\right)$. 
Indeed, $\zeta x_{k} \in \Omega_{n}$, which implies $\zeta x \in \Omega_{n}$ as $\zeta$ is continuous and $\Omega_{n}$ is closed. Also, we have $\zeta^{-1}(\zeta X)=X$ and therefore $x$ belongs to $\zeta^{-1}\left(\Omega_{n} \cap \zeta(X)\right)=\zeta^{-1}\left(\Pi_{n}\right)$. The proof is complete.

Proposition 3.1. Let $F: D(F) \subseteq Y \rightarrow Y^{*}$ be pseudo-monotone. Assume that $\zeta(X) \subseteq D(F)$. Then $F_{\zeta}(\cdot):=\zeta^{*} F \zeta(\cdot)$ is well defined and pseudo-monotone over the set $\zeta^{-1}(\Pi)$.

Proof. Let $y_{n}, y \in \zeta^{-1}(\Pi)$ be such that $y_{n} \rightarrow y$ as $n \rightarrow \infty$. In view of the assumption $\zeta(X) \subseteq D(F)$, we obtain $\zeta y_{n}, \zeta y \in D(F)$. Therefore $F_{\zeta}\left(y_{n}\right)$ and $F_{\zeta}(y)$ are well-defined. For the final assertion, we need to show that the convergence $y_{n} \rightarrow y$ as $n \rightarrow \infty$ along with

$$
\limsup _{n \rightarrow \infty}\left\langle F_{\zeta} y_{n}, y_{n}-y\right\rangle_{X} \leq 0
$$

implies that

$$
\liminf _{n \rightarrow \infty}\left\langle F_{\zeta} y_{n}, y_{n}-x\right\rangle_{X} \geq\left\langle F_{\zeta} y, y-x\right\rangle_{X}, \quad \forall x \in \zeta^{-1}(\Pi) .
$$

Since $\zeta$ is linear and continuous, from $y_{n} \rightarrow y$ as $n \rightarrow \infty$, we deduce that $\zeta y_{n} \rightarrow \zeta y$ as $n \rightarrow \infty$. Assume that (44) holds. This implies $\lim \sup \left\langle F z_{n}, z_{n}-z\right\rangle_{Y} \leq 0$, where $z_{n}=\zeta y_{n}, z=\zeta y$ such that $z_{n} \rightarrow z$ as $n \rightarrow \infty$. This in view of the pseudomonotonicity of $F$ yields

$$
\liminf _{n \rightarrow \infty}\left\langle F z_{n}, z_{n}-x\right\rangle_{Y} \geq\langle F z, z-x\rangle_{Y}, \quad \forall x \in D(F),
$$

and particularly

$$
\liminf _{n \rightarrow \infty}\left\langle F z_{n}, z_{n}-x\right\rangle_{Y} \geq\langle F z, z-x\rangle_{Y}, \quad \forall x \in \Pi .
$$

Consequently

$$
\liminf _{n \rightarrow \infty}\left\langle F_{\zeta} y_{n}, y_{n}-x\right\rangle_{X} \geq\left\langle F_{\zeta} y, y-x\right\rangle_{X}, \quad \forall x \in \zeta^{-1}(\Pi) .
$$

Therefore (5) holds. This completes the proof.

Proposition 3.2. Let $F: D(F) \subseteq Y \rightarrow Y^{*}$ and let $f \in Y^{*}$. Assume that $K_{1}, K_{2} \subset$ $Y$ such that $K_{1}$ is weakly dense in $K_{2}$. If there exists $u^{*} \in Y$ such that

$$
\left\langle F u^{*}-f, u-u^{*}\right\rangle_{Y} \geq 0, \quad \forall u \in K_{1},
$$

then (66) holds for all $u \in K_{2}$.

Proof. Let $\bar{u} \in K_{2}$ be arbitrary. Since $K_{1}$ is weakly dense in $K_{2}$, there exists a net $\left\{u_{i} \mid i \in I\right\} \subset K_{1}$ converging weakly to $\bar{u}$. Since $\left(F u^{*}-f\right) \in Y^{*},\left\langle F u^{*}-f, u_{i}-u^{*}\right\rangle_{Y}$ converges to $\left\langle F u^{*}-f, \bar{u}-u^{*}\right\rangle_{Y}$ and hence $\left\langle F u^{*}-f, \bar{u}-u^{*}\right\rangle_{Y} \geq 0$, the result follows.

Now we are in a position to discuss the solvability of $R V I(3)$.

Theorem 3.1. Let $n \in \mathbb{N}$ be arbitrary but fixed. Let $F$ be bounded, demicontinuous and pseudo-monotone with $\zeta(X) \subseteq D(F)$. Assume that there exists a strictly postive real $\alpha_{n} \leq \epsilon_{n}$ such that $\left\|F x-F_{n} x\right\|_{Y} \leq \alpha_{n} \kappa\left(\|x\|_{Y}\right), \quad \forall x \in \Pi_{n}$. Assume that there exist $y_{n} \in \Pi_{n}$ and a constant $\theta_{n}<\infty$ such that $\left\langle F x, x-\zeta y_{n}\right\rangle_{Y} \geq \theta_{n}\|x\|_{Y}$. Then $S_{n}(R V I) \neq \emptyset$. 
Proof. Let us consider an auxiliary $R V I$ (for short $A R V I$ ): find $x_{n} \in \zeta^{-1}\left(\Pi_{n}\right)$ such that

$$
\left\langle\zeta^{*} F \zeta x_{n}+\epsilon_{n} R x_{n}-\zeta^{*} f_{n}, z-x_{n}\right\rangle_{X} \geq 0, \quad \forall z \in \zeta^{-1}\left(\Pi_{n}\right) .
$$

Let $S_{n}(A R V I)$ be the set of all solutions to $A R V I(7)$. We first show that $S_{n}(A R V I)$ $\neq \emptyset$. Since $F$ is pseudo-monotone, it follows from Proposition 3.1 that $\zeta^{*} F \zeta$ is pseudo-monotone as well. The boundedness and the demicontinuity of $\zeta^{*} F \zeta$ is derived from the hypotheses on $\zeta$. In view of Theorem 2.1, it will suffice to show that $\left(\zeta^{*} F \zeta+\epsilon_{n} R\right)$ is coercive. For, we let $y \in \zeta^{-1}\left(\Pi_{n}\right)$ be arbitrary. We have

$$
\begin{gathered}
\left\langle\zeta^{*} F \zeta y+\epsilon_{n} R y, y-y_{n}\right\rangle_{X}=\left\langle F \zeta y, \zeta y-\zeta y_{n}\right\rangle_{Y}+\epsilon_{n}\left\langle R y, y-y_{n}\right\rangle_{X} \\
\geq \theta_{n}\|\zeta y\|_{Y}-\epsilon_{n}\left\|R y_{n}\right\|_{X}\left\|y-y_{n}\right\|_{X}+m\left\|y-y_{n}\right\|_{X}^{2},
\end{gathered}
$$

where the strong monotonicity of $R$ is used. From the above inequality it follows that

$$
\left\langle\zeta^{*} F \zeta y+\epsilon_{n} R y, y-y_{n}\right\rangle_{X} /\|y\|_{X} \rightarrow+\infty, \quad \text { as }\|y\|_{X} \rightarrow+\infty .
$$

Now it is a consequence of Theorem 2.1 that $S_{n}(A R V I) \neq \emptyset$.

Further, we claim that $S_{n}(A R V I) \subset S_{n}(R V I)$. For this we choose $y \in S_{n}(A R V I)$ arbitrarily. This implies that $y \in \zeta^{-1}\left(\Pi_{n}\right)$ and satisfies (7). Hence $\forall z \in \zeta^{-1}\left(\Pi_{n}\right)$ we have

$$
\begin{aligned}
\left\langle\zeta^{*} F_{n} \zeta y+\epsilon_{n} R y-\zeta^{*} f_{n}, z-y\right\rangle_{X} & \geq\left\langle\zeta^{*} F_{n} \zeta y-\zeta^{*} F \zeta y, z-y\right\rangle_{X} \\
& \geq-\left\|F_{n} \zeta y-F \zeta y\right\|_{Y}\|\zeta z-\zeta y\|_{Y} \\
& \geq-\alpha_{n} \kappa\left(\|\zeta y\|_{Y}\right)\|\zeta z-\zeta y\|_{Y} \\
& \geq-\epsilon_{n} \kappa\left(\|\zeta y\|_{Y}\right)\|\zeta z-\zeta y\|_{Y} .
\end{aligned}
$$

Thus $y \in S_{n}(R V I)$ and this confirms the inclusion $S_{n}(A R V I) \subset S_{n}(R V I)$. Since we have shown that $S_{n}(A R V I) \neq \emptyset$, we conclude that $S_{n}(R V I) \neq \emptyset$. This completes the proof.

We remark that the idea of introducing an error term in an RVI (cf. (3i)) was first given by Liskovets 12 for monotone operators. However, for monotone operators this approach has the disadvantage that an RVI with an error (cf. (31) term is not uniquely solvable, although an RVI without an error term (cf. (7)) is always uniquely solvable. Contrary to this, for pseudo-monotone operators, in general, neither (31) nor (7) has a singleton solution set.

For a sequence $\left\{s_{n}\right\}_{n=1}^{\infty}$, we represent by $\omega_{w}\left(s_{n}\right)$, the weak- $\omega$ limit set of $\left\{s_{n}\right\}_{n=1}^{\infty}$, that is, the set of all points $s$ such that it is always possible to extract a subsequence $\left\{s_{k}\right\}_{k=1}^{\infty}$ from $\left\{s_{n}\right\}_{n=1}^{\infty}$ satisfying $s_{k} \rightarrow s$ as $k \rightarrow \infty$.

Our first main result is as follows.

Theorem 3.2. Besides the hypotheses of Theorem 3.1, assume that $\zeta^{-1}\left(\Pi_{n}\right) \rightrightarrows$ $\zeta^{-1}(\Pi)$ and for $f_{n} \in Y^{*}$, there exists $\beta_{n}>0$ such that $\left\|f-f_{n}\right\|_{Y} \leq \beta_{n}$. Assume that $\epsilon_{n}, \alpha_{n}, \beta_{n}, \alpha_{n} / \epsilon_{n}, \beta_{n} / \epsilon_{n} \searrow 0$ as $n \rightarrow \infty$. Assume that $\left\{y_{n}\right\}_{n=1}^{\infty}$, where $y_{n} \in S_{n}(R V I)$ is chosen arbitrarily, be uniformly bounded. Then each $y \in \omega_{w}\left(y_{n}\right)$ is a solution to the following VI:

(8) find $w \in \zeta^{-1}(\Pi)$ such that $\left\langle\zeta^{*} F \zeta w-\zeta^{*} f, x-w\right\rangle_{X} \geq 0, \quad \forall x \in \zeta^{-1}(\Pi)$.

Furthermore, if $\Pi$ is weakly dense in $\Omega$, then $\zeta\left(\omega_{w}\left(y_{n}\right)\right) \subseteq S(V I)$. 
Proof. Since the space $Y$ is reflexive, the set $\omega_{w}\left(y_{n}\right)$ is nonempty. Let $y \in \omega_{w}\left(y_{n}\right)$ be arbitrary and let $\left\{y_{n}\right\}_{n=1}^{\infty}$ be a subsequence (we use the same notation for the subsequences as well) extracted from $\left\{y_{n}\right\}_{n=1}^{\infty}$ and satisfying $y_{n} \rightarrow y$ as $n \rightarrow \infty$. From the definition of $y_{n}$ it is clear that $y_{n} \in \zeta^{-1}\left(\Pi_{n}\right)$ and $\forall x \in \zeta^{-1}\left(\Pi_{n}\right)$ the following holds:

$$
\left\langle\zeta^{*} F_{n} \zeta y_{n}+\epsilon_{n} R y_{n}-\zeta^{*} f_{n}, x-y_{n}\right\rangle_{X} \geq-\epsilon_{n} \kappa\left(\left\|\zeta y_{n}\right\|_{Y}\right)\left\|\zeta x-\zeta y_{n}\right\|_{Y} .
$$

Since $\zeta^{-1}\left(\Pi_{n}\right) \rightrightarrows \zeta^{-1}(\Pi)$, it follows that $y \in \zeta^{-1}(\Pi)$ and there exists $\left\{z_{n}\right\}_{n=1}^{\infty}$ such that $z_{n} \rightarrow y$ with $z_{n} \in \zeta^{-1}\left(\Pi_{n}\right)$ (see Definition 2.3). On substituting $x=z_{n}$ in (9), we obtain

$$
\left\langle\zeta^{*} F_{n} \zeta y_{n}+\epsilon_{n} R y_{n}-\zeta^{*} f_{n}, z_{n}-y_{n}\right\rangle_{X} \geq-\epsilon_{n} \kappa\left(\left\|\zeta y_{n}\right\|_{Y}\right)\left\|\zeta z_{n}-\zeta y_{n}\right\|_{Y} .
$$

This implies

$$
\begin{aligned}
& \limsup _{n \rightarrow \infty}\left\langle\zeta^{*} F \zeta y_{n}, y_{n}-z_{n}\right\rangle_{X} \\
& \leq \limsup _{n \rightarrow \infty}\left\langle f_{n}-f, \zeta y_{n}-\zeta z_{n}\right\rangle_{Y}+\limsup _{n \rightarrow \infty}\left\langle f, \zeta y_{n}-\zeta z_{n}\right\rangle_{Y} \\
&+\limsup _{n \rightarrow \infty}\left\langle\epsilon_{n} R y_{n}, z_{n}-y_{n}\right\rangle_{X}+\epsilon_{n} \kappa\left(\left\|\zeta y_{n}\right\|_{Y}\right)\left\|\zeta z_{n}-\zeta y_{n}\right\|_{Y} \\
&+\limsup _{n \rightarrow \infty}\left\langle F \zeta y_{n}-F_{n} \zeta y_{n}, \zeta y_{n}-\zeta z_{n}\right\rangle_{Y} \\
& \leq \limsup _{n \rightarrow \infty} \beta_{n}\left[\left\|\zeta z_{n}-\zeta y\right\|_{Y}+\left\|\zeta y_{n}-\zeta y\right\|_{Y}\right] \\
&+\limsup _{n \rightarrow \infty}\left[\|f\|_{Y}\left\|\zeta z_{n}-\zeta y\right\|_{Y}+\left\langle f, \zeta y_{n}-\zeta y\right\rangle_{Y}\right] \\
&+\limsup _{n \rightarrow \infty} \epsilon_{n}\left\|R y_{n}\right\| X\left\|z_{n}-y_{n}\right\|_{X} \\
&+\limsup _{n \rightarrow \infty}\left(\alpha_{n}+\epsilon_{n}\right) \kappa\left(\left\|\zeta y_{n}\right\|_{Y}\right)\left\|\zeta y_{n}-\zeta z_{n}\right\|_{Y}
\end{aligned}
$$

Since $\left\{\epsilon_{n}, \alpha_{n} \beta_{n},\left\langle f, \zeta y_{n}-\zeta y\right\rangle,\left\|\zeta z_{n}-\zeta y\right\|\right\} \rightarrow 0$ as $n \rightarrow \infty$ and the remaining terms are bounded, we deduce that $\limsup _{n \rightarrow \infty}\left\langle\zeta^{*} F \zeta y_{n}, y_{n}-z_{n}\right\rangle_{X} \leq 0$ and accordingly

$$
\begin{aligned}
\limsup _{n \rightarrow \infty}\left\langle\zeta^{*} F \zeta y_{n}, y_{n}-y\right\rangle_{X} & \leq \limsup _{n \rightarrow \infty}\left\langle\zeta^{*} F \zeta y_{n}, z_{n}-y\right\rangle_{X} \\
& =0 .
\end{aligned}
$$

In view of the pseudo-monotonicity of $F$ and Proposition 3.1, we obtain

$$
\liminf _{n \rightarrow \infty}\left\langle\zeta^{*} F \zeta y_{n}, y_{n}-w\right\rangle_{X} \geq\left\langle\zeta^{*} F \zeta y, y-w\right\rangle_{X}, \quad \forall w \in \zeta^{-1}(\Pi) .
$$

From $\left\langle\zeta^{*} f, y_{n}-w\right\rangle_{X} \rightarrow\left\langle\zeta^{*} f, y-w\right\rangle_{X}$ as $n \rightarrow \infty$ and the above inequality we deduce that

$$
\liminf _{n \rightarrow \infty}\left\langle\zeta^{*} F \zeta y_{n}-\zeta^{*} f, y_{n}-w\right\rangle_{X} \geq\left\langle\zeta^{*} F \zeta y-\zeta^{*} f, y-w\right\rangle_{X} \quad \forall w \in \zeta^{-1}(\Pi)
$$

Let $z \in \zeta^{-1}(\Pi)$ be arbitrary and let $z_{n} \in \zeta^{-1}\left(\Pi_{n}\right)$ be such that $z_{n} \rightarrow z$ as $n \rightarrow \infty$. In order to get an estimate for the term appearing on the right side of (13), we 
consider

$$
\begin{aligned}
& \liminf _{n \rightarrow \infty}\left\langle\zeta^{*} F \zeta y_{n}-\zeta^{*} f, y_{n}-z\right\rangle_{X} \\
& \leq \limsup _{n \rightarrow \infty}\left\langle\zeta^{*} F \zeta y_{n}-\zeta^{*} f, y_{n}-z\right\rangle_{X} \\
& \leq \limsup _{n \rightarrow \infty}\left\langle\zeta^{*} F \zeta y_{n}-\zeta^{*} f, y_{n}-z_{n}\right\rangle_{X} \\
&+\limsup _{n \rightarrow \infty}\left\langle\zeta^{*} F \zeta y_{n}-\zeta^{*} f, z_{n}-z\right\rangle_{X} \\
& \leq \limsup _{n \rightarrow \infty}\left\langle\zeta^{*} F \zeta y_{n}-\zeta^{*} F_{n} \zeta y_{n}, y_{n}-z_{n}\right\rangle_{X} \\
&+\limsup _{n \rightarrow \infty}\left\langle\zeta^{*} F_{n} \zeta y_{n}-\zeta^{*} f_{n}, y_{n}-z_{n}\right\rangle_{X} \\
&+\limsup _{n \rightarrow \infty}\left\langle\zeta^{*} f_{n}-\zeta^{*} f, y_{n}-z_{n}\right\rangle_{X} \\
& \leq \limsup _{n \rightarrow \infty} \alpha_{n} \kappa\left(\left\|\zeta y_{n}\right\|_{Y}\right)\left\|\zeta y_{n}-\zeta z_{n}\right\|_{Y} \\
&+\limsup _{n \rightarrow \infty}\left\langle\zeta^{*} F_{n} \zeta y_{n}-\zeta^{*} f_{n}, y_{n}-z_{n}\right\rangle_{X} \\
&+\limsup _{n \rightarrow \infty} \beta_{n}\left\|\zeta y_{n}-\zeta z_{n}\right\| \\
& \leq \limsup _{n \rightarrow \infty}\left\langle\zeta^{*} F_{n} \zeta y_{n}-\zeta^{*} f_{n}, y_{n}-z_{n}\right\rangle_{X} \\
& \leq \limsup _{n \rightarrow \infty} \epsilon_{n}\left(\left\langle R y_{n}, z_{n}-y_{n}\right\rangle_{X}+\kappa\left(\left\|\zeta y_{n}\right\|_{Y}\right)\left\|\zeta y_{n}-\zeta z_{n}\right\|_{Y}\right) \\
&= 0,
\end{aligned}
$$

where the second last inequality is a consequence of (10).

The above inequality when combined with (13) yields

$$
\left\langle\zeta^{*} F \zeta y-\zeta^{*} f, z-y\right\rangle_{X} \geq 0, \quad \forall z \in \zeta^{-1}(\Pi) .
$$

This proves the first assertion. For the second one, set $\zeta y:=u^{*}$. From (15) we obtain

$$
\left\langle F u^{*}-f, u-u^{*}\right\rangle_{Y} \geq 0, \quad \forall u \in \Pi .
$$

An application of Proposition 3.2 brings us to the conclusion that the above inequality is valid for all $u \in \Omega$. Therefore $\zeta y:=u^{*}$ satisfies

$$
\left\langle F u^{*}-f, u-u^{*}\right\rangle_{Y} \geq 0, \quad \forall u \in \Omega .
$$

Consequently $\zeta y \in S(V I)$. This completes the proof.

Observe that (8) is the embedded form of (11). The above proof makes it clear that the solvability of (10) is implied from the solvability of (8). Therefore, in the present approach, first the perturbed $V I$ is embedded into $X$ and then by the regularization procedure the embedded version of (1) is recovered.

In order to prove the strong convergence we need to recall that, given a real reflexive Banach space $Z$ with $Z^{*}$ as its topological dual, an operator $T: Z \rightarrow Z^{*}$ is said to satisfy condition $S$ if $x_{n} \rightarrow x$, together with $\lim \sup \left\langle T x_{n}, x_{n}-x\right\rangle=0$, implies that $x_{n} \rightarrow x$.

Theorem 3.3. Besides the hypotheses of Theorem 3.2 assume that either $\zeta^{*} F \zeta$ satifies the condition $S$ or for each $\left\{z_{n}\right\}_{n=1}^{\infty}$ such that $z_{n} \rightarrow z$, we have that $\lim _{n \rightarrow \infty}\left\langle R z_{n}, z_{n}-z\right\rangle=0$. Then for $y \in \omega_{w}\left(y_{n}\right)$, there is a subsequence of $y_{n}$ converging strongly to $y$. 
Proof. Proceeding as in the proof of Theorem 3.2, we can show that for a subsequence $\left\{y_{n}\right\}_{n=1}^{\infty}$ such that $y_{n} \rightarrow y$ as $n \rightarrow \infty$, the following relation holds (cf. (12)):

$$
\limsup _{n \rightarrow \infty}\left\langle\zeta^{*} F \zeta y_{n}, y_{n}-y\right\rangle_{X} \leq 0 .
$$

An analogue of (13) with $w=y$ reads as

$$
\liminf _{n \rightarrow \infty}\left\langle\zeta^{*} F \zeta y_{n}, y_{n}-y\right\rangle \geq 0 .
$$

A combination of the above two inequalities yields

$$
\left\langle\zeta^{*} F \zeta y_{n}, y_{n}-y\right\rangle \rightarrow 0 \quad \text { as } n \rightarrow \infty .
$$

Therefore, if $\zeta^{*} F \zeta$ satisfies the condition $S$, we obtain the desired strong convergence. Otherwise, since $y_{n} \rightarrow y$ as $n \rightarrow \infty$, by the assumption we have $\lim _{n \rightarrow \infty}\left\langle R y_{n}, y_{n}-y\right\rangle_{X}=0$. Since the condition $S$ is always satisfied by strongly monotone operators, we deduce from the above that $y_{n} \rightarrow y$ as $n \rightarrow \infty$. This completes the proof.

We now briefly touch upon the possibility of some applications of the results of this paper.

Let $K$ be a nonempty, closed and convex subset of a real Banach space $Z$. Given a functional $A: Z \rightarrow \Re$, consider the following minimization problem: find $x \in K$ such that

$$
A(x) \leq A(z), \quad \forall z \in K .
$$

If $A$ is convex and Gâteaux differentiable, then (17) is identical to the following $V I$ :

$$
\text { find } x \in K \text { such that }\langle\nabla A(x), z-x\rangle \geq 0, \quad \forall z \in K,
$$

where $\nabla A$ is the Gâteaux derivative of $F$. Let $B$ be a Banach space which is embedded into $Z$, that is, there exists a linear subspace $V \subseteq Z$ such that $I(B) \equiv V$. Here $I$ is the embedding map. For the sake of simplicity, assume that $K \subseteq V$.

Clearly the above minimization problem is the framework of the present work under the setting $K:=\Omega, Z:=Y, B:=X$, and $I:=\zeta$. The density assumption is now superfluous because of the condition that $K \subseteq V$.

A few examples of the function spaces satisfying the above requirements are as follows.

Example 3.1. Let $Z=W^{m, p}(G)$ and $B=W^{s, 2}(G)$, where $s>m+(n / 2)-(n / p)$ and $G \subset \Re^{n}$. In this case $I$ is compact. Here $W^{m, p}(G)$ and $W^{s, 2}(G)$ are the familiar Sobolev spaces. For the details, the reader is referred to Zeidler [20].

Example 3.2. Let $Z=B V(G)$ and $B=W^{1,2}(G)$. In this case $I$ is only continuous. Here $B V(G)$ represents the space of functions of bounded variations. For details, the reader is referred to Nashed-Scherzer [16].

Example 3.3. Let $E$ be an embeddable space, $E^{*}$ be the continuous dual of $E$ and $H$ be the corresponding Hilbert space, that is, among other properties the relation $E \subset H$ (in the usual sense of embedding) holds. For the definition of embeddable spaces and various examples including general Banach spaces and topological spaces, the reader is referred to Vainberg [19]. It is well known that for embeddable space $E$, the relation $H \subset E^{*}$ is true. In this case $B=H$ and $Z=E^{*}$ serve our purpose. 
A general approach of construction of the mapping $\zeta$ can be based on the square roots of certain operators. We illustrate this by giving some examples in the following. First, we mention that the methodology adopted in these examples has its origin in solving the Hammerstein equation by the use of the variational methods (cf. [19], [20]).

Example 3.4. Let the linear integral operator $\mathcal{J}: L^{q}(S) \rightarrow L^{p}(S)\left(p \geq 2, p^{-1}+\right.$ $\left.q^{-1}=1, \operatorname{mes}(S)<\infty\right)$ defined by

$$
\mathcal{J}(v):=\int_{S} K(x, y) v(y) d y
$$

be positive and selfadjoint (see [19] for details). Then $\mathcal{J}=T^{*} T$ where $T^{*}:=U$ is the positive square root of the restriction $\mathcal{J}_{L^{2}(S)}$ of $\mathcal{J}$ to $L^{2}(S)$. In the present case we have $T=T^{* *}=U^{*}$, where $U: L^{2} \rightarrow L^{p}$ and $U^{*}: L^{q} \rightarrow L^{2}$. By setting $X:=L^{2}(S)$ and $Y:=L^{p}(S)$ and by choosing $\zeta:=U$ we are in the framework of our results.

In fact the considerations of the above example make sense in more general Orlicz spaces as shown in our next example. First, we recall that for given $M(\cdot)$ and $N(\cdot)$ complementary young functions and the set $L_{M}^{\alpha}:=\left\{u(x): \int_{S} M(\alpha|u(x)|) d x<\right.$ $+\infty\}$, where $\alpha>0, S$ is as above and $u(x)$ is measurable on $S$, the Orlicz space $L^{M}$ is defined as the union of the clasees $L_{M}^{\alpha}$ for all $\alpha>0$ (see [19] Chapter VIII] for details). The space $L^{N}$ is defined analogously and, under suitable conditions, $L^{M}$ serves as the dual of $L^{N}$. We shall denote by $L_{M}^{f}$ the intersection of all the classes $L_{M}^{\alpha}$.

Example 3.5. Consider a linear integral operator $\mathcal{J}$ of the form given in Example 3.4. Assume that $\mathcal{J}$ is positive and maps all of $L^{N}$ into $L_{M}^{f}$. Then $\mathcal{J}$ is bounded from $L^{N}$ to $L_{M}^{f}$ and its restriction $\mathcal{J}_{0}$ to $L^{2}(S)$ is also a bounded operator. Furthermore, it can be represented as $\mathcal{J}=U_{0} U$, where $U_{0}=\mathcal{J}_{0}^{1 / 2}: L^{2}(S) \rightarrow L^{M}$ and $U$ is a continuous extension of $U_{0}$ from $L^{N}$ to $L^{2}(S)$. Moreover $\left\langle w, U_{0}(v)\right\rangle=\langle U(w),(v)\rangle$ for $v \in L^{2}, w \in L^{N}$, where $\left\langle w, U_{0}(v)\right\rangle=\int_{S} w(x) U_{0} v(x) d x$. Now our results are extendable to the case when $X:=L^{2}(S), Y:=L^{M}$ and $\zeta:=U_{0}$.

In order to throw some light on the representation of $\zeta$ and $\zeta^{*}$ in the above two examples, we briefly recall some facts about the square root of linear integral operators. Let $\mathcal{J}: L^{q}(S) \rightarrow L^{p}(S)\left(p>2, p^{-1}+q^{-1}=1, \quad S \subset \mathbb{R}^{n}, \operatorname{mes}(S)<\infty\right)$ be a completely continuous integral operator with a positive selfadjoint restriction $\mathcal{J}_{L^{2}(S)}$ of $\mathcal{J}$ to $L^{2}(S)$ which can be expressed as (see [19, Chapter V]) $\mathcal{J}_{L^{2}(S)}(x)=$ $\sum_{k=1}^{\infty} \frac{\left\langle x, f_{k}\right\rangle}{\lambda_{k}} f_{k}\left(x \in L^{2}(S), f_{k} \in L^{2}, \lambda_{k}>0\right)$, where $f_{k}$ are the eigenfunctions of $\mathcal{J}_{L^{2}(S)}$ corresponding to the eigenvalues $\lambda_{k}$. Then for each $k, f_{k} \in L^{p}$, and the square root $\mathcal{J}_{L^{2}(S)}^{1 / 2}$ has a continuous extension $U^{*}: L^{q}(S) \rightarrow L^{2}$ which can be written as $U^{*}(v)=\sum_{k=1}^{\infty} \frac{\left\langle v, f_{k}\right\rangle}{\sqrt{\lambda_{k}}} f_{k}\left(v \in L^{q}(S)\right)$ and its adjoint $U^{* *}$ as $U^{* *}(u)=\sum_{k=1}^{\infty} \frac{\left\langle u, f_{k}\right\rangle}{\sqrt{\lambda_{k}}} f_{k}$ $\left(u \in L^{2}(S)\right)$.

\section{Concluding Remarks}

In this paper we have considered a stable approximation of the variational inequalities with pseudo-monotone operators. The variational inequality is first embedded into a real reflexive Banach space and then, by making use of the reflexive nature of the space, weak as well as strong approximation is established. 
Here we have worked under the assumption that the stabilized solutions are uniformly bounded. Further, we have used this to prove the solvability of the original problem. It will be interesting to study the converse as well. It is wellknown that for monotone operators the converse holds. Recently Gwinner [6] has shown that for pseudo-monotone operators the converse holds under additional conditions on the operator.

\section{ACKNOWLEDGMENTS}

The authors are grateful to the referee for a careful reading and helpful remarks.

\section{REFERENCES}

1. Y. I. Alber, A. D. Butnariu and I. Ryazantseva: Regularization methods for ill-posed inclusions and variational inequalities with domain perturbations, J. Nonlinear Convex Anal., Vol. 2, pp. 53-79 (2001). MR 2002d:47087

2. F. E. Browder and B. A. Ton: Nonlinear functional equations in Banach spaces and elliptic super regularization, Math. Zeitschr., Vol. 105, pp. 177-195 (1968). MR 38:582

3. B. Djafari Rouhani, E. Tarafdar and P. J. Watson: Fixed point theorems, coincidence theorems and variational inequalities, Lecture Notes in Economics and Mathematical Systems, Vol. 502, pp. 183-188, Springer, Berlin, 2001. MR 2002b:47129

4. F. Giannessi: Embedding variational inequalities and their generalizations into a separation scheme, J. Inequal. Appl. 1, pp. 139-147 (1997). MR 2000j:49017

5. F. Giannessi and A. A. Khan: Regularization of non-coercive quasi variational inequalities, Control and Cybernetics, Vol. 29, pp. 91-110 (2000). MR 2001f:49019

6. J. Gwinner: A note on pseudomonotone functions, regularization, and relaxed coerciveness, Nonlinear Analysis, Vol. 30, pp. 4217-4227 (1997).

7. P. T. Harker and J.S. Pang: Finite dimensional variational inequality and complementarity problems; A survey of theory algorithms and applications, Mathematical Programming, Vol. 48, pp. 161-220 (1990). MR 91g:90166

8. G. Isac: Tikhonov regularization and the complementarity problem in Hilbert spaces, J. Math. Anal. Appl., Vol. 174, pp. 53-66 (1993). MR 94b:49014

9. V. V. Kalashnikov and A. A. Khan: A regularization approach for variational inequalities with pseudo-monotone operators, Inderfurth, K. (ed.) et al., Operations research proceedings 1999, pp. 19-22, Springer, Berlin, 2000.

10. A. A. Khan: A regularization approach for variational inequalities, Comput. Math. Appl., Vol. 42, pp. 65-74 (2001). MR 2002b:49020

11. D. Kinderlehrer and G. Stampacchia: An introduction to variational inequalities and their applications, Academic Press, New York, 1980. MR 81g:49013

12. O. A. Liskovets: Regularization of problems with discontinuous monotone, arbitrarily perturbed operators, Soviet Math. Dokl., Vol. 28, pp. 324-327 (1983). MR 85e:47092

13. O. A. Liskovets: Regularization of ill-posed mixed variational inequalities, Sov. Math. Dokl., Vol. 43, pp. 384-387 (1991). MR 92i:47085

14. U. Mosco: Convergence of convex sets and of solutions of variational inequalities, Adv. Math. Vol. 3, pp. 510-585 (1969). MR 45:7560

15. M. Z. Nashed and F. Liu: On nonlinear ill-posed problems II: Monotone operator equations and monotone variational inequalities, Lecture Notes in Pure and Applied Mathematics, Vol. 178, pp. 223-240, Marcel Dekker, Inc. New York, 1996. MR 97e:47115

16. M. Z. Nashed and O. Scherzer: Stable approximation of nondifferentiable optimization problems with variational inequalities, Contemporary Mathematics, Vol. 204, pp. 155-170 (1997). MR 98j:49015

17. M. A. Noor, K. I. Noor and T. M. Rassias: Some aspects of variational inequalities, J. Comput. Appl. Math. 47, 285-312 (1993). MR 94h:49017

18. B. A. Ton: Nonlinear operators on convex subsets of Banach spaces, Math. Ann., Vol. 181, pp. 35-44 (1969). MR 39:4717 
19. M. M. Vainberg: Variational methods and method of monotone operators in the theory of nonlinear equations, John Wiley, New York, 1973. MR 57:7286b

20. E. Zeidler: Nonlinear Functional Analysis and its Applications, Vol. II/B, Springer-Verlag, New York, 1990. MR 91b:47002

Institute for Studies in Nonlinear Analysis, School of Mathematical Sciences, Shahid Beheshti University, P.O. Box 19395-4716 Evin, 19834 Tehran, Iran

E-mail address: b-rohani@cc.sbu.ac.ir

Institute of Applied Mathematics, University of Erlangen-Nürnberg, Martensstr. 3, 91058 Erlangen, Germany

E-mail address: khan@am.uni-erlangen.de

Current address: Department of Mathematical Sciences, Michigan Technological University, 319 Fisher Hall, 1400 Townsend Drive, Houghton, Michigan 49931-1295

E-mail address: aakhan@mtu.edu 